\section{H.F. KAVEH,}

B. DEFFONTAINES,

B. FRUNEAU

Université Paris-Est Laboratoire Géomaterieux et Géologie de l'ingénieur (G2i) 5, bd Descartes

Champs-sur-Marne -77454 Mame-la-Vallée Cedex 2 frederic.kaveh@univ-rnlv.f. benoit. deffontaines@univ-mlv.ff benedicte.fruneau@univ-mlv.fr

\section{Apports d'un Système} d'Information Géographique et de la télédétection pour l'étude des mouvements de terrain induits par l'aléa sécheresse : applications à l'Est de l'île-de-France
Quels sont les apports de la tèlédétection (optique et radarj pour détecter et mesurer les déformations induites par l'aléa sécheresse et plus particulièrement le retrait/gonflement des sols argileux dans l'Est de l'İlede-France? Lintégration des données dans un Système d'Information Géographique devrait favoriser l'analyse des principaux facteurs à l'origine de ces déformations. Ainsi un modèle numérique de terrain (BdAltio de I'TGN) permet d'extraire les pentes topographiques, l'imagerie aérienne (photographie ortho rectif́ée de l'IGN) révẻle l'occupation du sol, et l'application de la méthode d'interférométrie radar DInSAR favorise la localisation, la caractérisation et la quantification des déformations. D'autres données numériques sont combinées, telles une cartographie détaillée du mode d'occupation du sol de l'LAUHIF et la cartographie géologique du BRGM, qui permet de localiser des affieurements d'argiles gonflantes. Ce travail est compare à l'approche cartographique de l'aléa du retrait/gonflement des sols argileux effectuếe par le BRGM. En conclusion, la nature gonflante du sol, le type de tissu urbain. la topographie ou encore la présence de végétation arborée par exemple, sont autant d'éléments quí expliquent localement la sinistralité dans l'Est de l'lle-de-France.

Mots-clés : sécheresse géotechnique, retrait/gonflement, argile, interférométrie, SIG, Est parisien.
NDLR : Les discussions sur cet articie sont acceptées jusqu'au 30 avil 2008.
What are the remote sensing (optical and radar wavelength) contributions to locate, characterize and quantify the deformations issued from the dryness hazard in the eastern part of the Paris basin? The data integration within a GIS helps to analyse the major parameters which induce such Jow deformations. So a Digital Terrain Model (DTM) from BdAlti of IGN permits the extraction of the topographic slopes, the 
orthophotography (aerial photograph orthorectified) reveals the land use, and the Differential Interferomety DInSAR gives inctication in coherent areas to locate, characterize and guantify the low deformations. Other data are superimposed such as the geologic map issued from BRGM mapping which helps to locate the outcropping clays submitted to the phenomena, and IAURIF map which helps to better characterize the land occupation. This work is compared to the ane done by the BRGM. To conclude the presence of clays sensible to hygrometry, the type of land cccupation such as the presence of trees is key points to explain the disorders that exist in the eastern Paris basir.

Key words: drought hazard, swelling clays, interferonetry, GIS, East Paris basin.

\section{Introduction}

La sêcheresse est à l'ongine de nombreux mouvements de la surface du sol. Le phénomène de retrait/ gonflement d'Argile (RGA ci-après) exacerbe ces mouvements dans les sols où des argiles sont présentes. Dure et cassante lorsqu'elle est desséchée, l'argile devient plastique et malléable à partir d'un certain niveau d'humidité. Les déplacements induits sont de faible amplitude en valeur absolue (du mm à $15 \mathrm{~cm}$ dans la zone d'étude), mais le phénomène de tassement différentiel est à l'origine d'importants dommages sur les battiments. Le but de ce travail est de mieux comprendre les facteurs à l'origine des déformations de la surface topographique urbanisée induite par le RGA en utilisant les donnêes de têlédétection optique et radar, intégrées au sein d'un Systène d'Tnformetion Géographique (SIG). Les données optiques sont des orthophotos géoréférencées et les données radars utilisées sont des interférogrammes obtenus par la méthode interféronétrique différentielle DInSAR'. En cuise d'application, la région lle-de-France a été choisie car particulièrement affectẻe par le phénomène avec un cumul de 559 communes sinistrées sur 1300 (arrêté ministériel du 18/09/1998) pour les périodes de sécheresse de 1989-1992 et de 1996-1997, représentant une sinistralité de $43 \%$. En particulier, deux dêpartements de l'Est parisien, le Val-de-Marne (94) et la Seine-Sainl-Denis (93) ont les taux de sinistralité parmi les plus élevés de France avec le second et le troisième rang respectivement, selon la Caisse centrale de réassurance. En effet, le fort taux d"urbanisation et le caractère pavilionnaire de leurs zones résidentielles situées sur des argles gonflantes font de ces départerments des territoires vilnérables au RGA. Plus récernment, la canicule de 2003 et la sécheresse de l"année 2005 sourlignent la récurrence du phénomène, dans le contexte de réchauffement climatique actuel selon l'étude de Hensen et al. (2006).

La compréhension du phénomène ainsi que sa cartographle est un objet de recherche d'actualité. D'après Vincent (2006), la délimitation des zones sensibles est la première étape d'une politique de prévention des risques. Elle permet d'appliquer des normes de construction plus sévères ou d'imposer des aménagements adaptés dans les zones à risque. Un premier travail d'inventaire des zones concernées par l'aléa RGA a été réalisé par le Burcau de recherche géologique et minière (BRGM) à l'échelle départementale en se basant sur la cartographie de la lithologie, la sinistralité et des paramètres géotechniques des formations issus des cartes géologiques au 1/50 000. A la demande du ministère de l'Écologie et du Développement durable (MEDD), ces travaux ont été transposés à l'échelle de la commune, afin d'établir des plans de prévention de risque. Cependant, compte tenu des incertitudes sur la lithologie des sols sub-aflleurants et de la grande variabilité dans leur composition minéralogique, la cartographie de l'aléa réalisée par le BRGM reste imprécise à l'échelle d’un quartier d'habitation.

Localement, les analyses géotechniques du sol permettent de caractériser précisément le potentiel expansif du sol (Bigot et al., 2000). Cependant, les essais en laboratoire ont un coût et sont long a effectuer. Il est donc difficile de multiplier les échantillonnages pour un terrain donné et de couvrir ainsi une vaste zone géographique. La télédétection, en tant qu'outil de mesure directe à distance, permettrait d'avoir une vue synoptique du phénomène et pourrait être utilisée pour suivre dans le temps les déplacements induits et de préciser localement lorigine des désordres induits par la sécheresse. Les synthèses de Van der Meer (1999) et de Kariuki et al. (2004) exposent succinctement les méthodes et les techniques en télédétection optique utiles à la problématique du RGA. En télédétection optique, l'imagerie hyper spectrale a déjà permis de caractériser la minéralogie des sols affleurants (Chabillat et al., 2002 ; Ben-Dor et al., 2002 ; Kariuki et Van der Meer, 2003). Cependant, si de bons résultats sont obtenlis pour des milieux arides, faiblements végétalisés et peu urbanisés, la télédétection optique reste inadaptée en ile-de-France de par la forte urbanisation et l'abondance de la végétation.

Dans le domaine de la télédétection radar, l'interférométrie différentielle radar permet dans certaines conditions de mesurer des déformations discrètes de faible amplitude avec, au mieux, une précision miltimétrique, (Massonnet et Feigl, 1998). Cependant, à notre connaissance, cette technique n'a pas encore été appliquée aux mouvements induits par la sécheresse. L'objet de cette étude est donc d'évaluer la faisabilité de la technique DInSAR pour l'étude des effets de la sécheresse géotechnique en vue de localiser, caractériser et quantifier les déformations de la surface du sol. Les résultats et les limites de cette technique appliquée au suivi du phénomène RGA sont précisés ci-dessous. Les traitements ont été réalisés avec des données d'archives du satelltte ERS acquises entre les années 1991 et 2000, en particulier pour la sécheresse de 1996/1997. sur l'Est parisien. Après avoir exposé le phënomène RGA et les facteurs participants, la méthode interférométrique utilisée sera détaillée. On présentera les résultats d'une analyse etfectuée à partir de l'ímagerie aérienne, des cartes géologiques et de la cartographie 
de l'aléa réalisée par le BRGM, des cartes de pente et enfin des interférogrammes choisis sur les périodes de sécheresse étudiées.

\section{2}

\section{Le retrait/gonflement d'Argile ( $R G A)$}

\section{1}

\section{Le risque RGA}

La définition du phénomène de retrait/gonflement des sols argileux en terme d'aléa ou de risque est importante tant les facteurs environnementaux qui interviennent dans la problématique sont nombreux. Rappelons qu'un aléa se définit par la probabilité qu'un évènement naturel se produise. Le risque est l'intersection de cet aléa avec un enjeu ou avec une vulnérabilité (Bourrelier et al., 2000) et se mesure par une intensité. La détection des mouvements de la surface du sol par l'interférométrie radar implique gue l'on étudie les effets de la sécheresse en tant qu'aléa, qui croisé avec la susceptibilitế du sol au retrait/gonflement donne la sécheresse géotechnique (Fig. 1a). Cependant la problématique fait aussi intervenir un enjeu, tel que les zones urbaines mais aussi la vilnérabilité des båtiments et les informations relatives à leurs dommages. Le retrait/gonflement des sols argileux est donc un risque, puisqu'il résulte de la combinaison de l'aléa sécheresse, de la susceptibilité du sol au retraì/ gonflement et de la vulnérabilité des infrastructures (Fig. 1b).

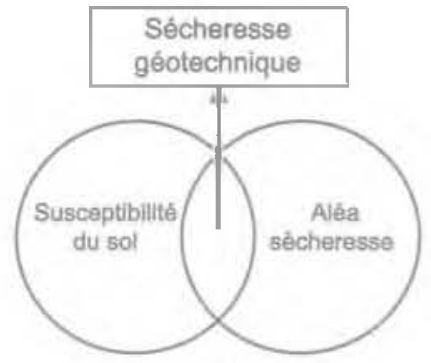

(a)

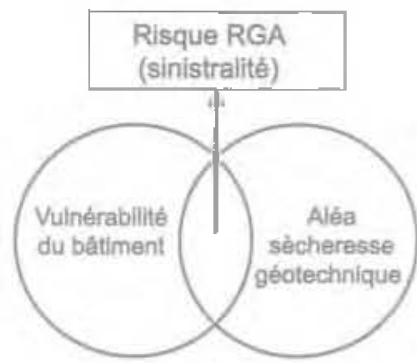

(b)
月ธ. 1 Schémas risque/aléa appliquésàla sécheresse géotechnique. En (a), Yaléa sëcheresse géotechnique résulte de l'aléa sécheresse et de la susceptibilitë du sol et en (b), le risque du RG.A résulte de la vulnérabilité des bâtiments et de l'aléa sécheresse géotechnique. Scheme Risk/hazard applied to geotechnical drought.

(a) The geotectrical drought hazard.

(b) the swelling soils risk.

\section{Le phénomène physique}

Le cycle de tassement et du retour à l'équilibre d'un sol est un phénomène physique naturel qui se traduit par une variation de volume du sol, qui est étroitement liée à la variation de la teneur en eau. D'un point de vue granulométrique, la plupart des types de sols voient leur volume évoluer selon le retrait ou l'apport d'eau.

Mais ce sont les propriétés minéralogiques des argiles qui font la particularité du phénomène de RGA : la surface spécifícue élevée des feuillets argileux permet aux particules d'argile d'adsorber une quantité importante d'eau. Le potentiel de retrait/gonflement est ainsi particulièrement élevé pour Ies minéraux argileux appartenant au groupe des smectites (en particulier la montmorillonite) et, dans une moindre mesure, au groupe des interstratifiés, caractérisé par l'alternance plus ou moins régulière de feuillets de natures différentes tels que les smectites/ilites.

Quel est l'ordre de grandeur de ces mouvements susceptibles d'être détectés par la technique d'interférométrie radar? Lors d'une sécheresse, la tranche la plus superficielle du sol argileux est soumise à l'évaporation sur plusieurs mètres de profondeur. Il en résulte un tassement vertical et l'ouverture de fissures vertica. les superficielles. L'amplitude des mouvements verticaux est d'ordre centimétrique et peut dépasser $10 \mathrm{~cm}$ en certaines circonstances. Une couche argileuse saturée de un mètre d'épaisseur qui subirait une diminution relative de la teneur en eau de $10 \%$, génêrerait un tassement d"environ cing centimètres (Barbier, 2004). Ces variations de volume, surtout lorsqu'elle donnent lieu à des tassement différentiels, ont des conséquences importantes sur l'état des bâtiments, en particulier sur ceux dont la conception n'est pas adaptée pour résister à ce type de mouvement.

\section{3}

\section{Les facteurs}

Pour dêcrire les facteurs influençant l'aléa RGA. on distingue les facteurs de prédisposition et les facteur's de déclenchement (Donsimoni et al., 2003). Cette nomenclature proposée par le BRGM a été reprise dans nos travaux.

Les facteurs de prédisposition sont ceux dont la présence fait que le phénomène de retrait-gonflement est possible, mais ne suffisent pas à eux seuis pour le déclencher. Ces facteurs sont fixes ou évoluent très lentement avec le temps. Ce sont : (1) la nature du sol, (2) le contexte hydrogéologique, (3) la présence d'une nappe phréatique, (4) le contexte géomorphologique el (5) les défauts de construction.

Les facteurs de déclenchement sont ceux dont la présence provoque le phénomène de retrait-gonflement, mais qui n'ont d'effet significatif que s'il existe des facteurs de prédisposition préalables. Ce sont : (1) les conditions climatiques, (2) les facteurs anthropiques et (3) la végétation.

Ces éléments doivent donc être théoriquement pris en compte lors d'une étude complète de l'impact d'une sécheresse. En effet, selon le processus considéré, l'extension spatiale du mouvement liée aux effets de la sécheresse est variable : la déformation de la surface du sol peut être très localisée pour le retrait/gonflement de sols argileux, mais aussi plus étendue dans le cas d'une déstabilisation des pentes ou dans le cas d'un tassement d'une formation géologique liê au battement du niveau de la nappe d'eau souterraine. Les facteurs de prédisposition selon le contexte environnant, comme la présence de végétation ou l'existence d'une nappe aquifère temporaire peuvent certainement modifier de manière relative l'extension spatiale de ces mouvements. Leurs influences doivent être prises en 
compte pour mieux décrire les signaux détectés dans les interférogrammes calculés.

\section{4}

\section{Les périodes relatives de sécheresse}

La détermination des pếriodes de sécheresse est un travail nécessaire pour l'étude des effets de l'aléa sécheresse sur le sol par interférométrie radar. Connầtre ces périodes permet de sélectionner les données radar utiles et donne des reperes temporels pratiques lors de l'interprétation des interférogrammes. La détermination d'une période de sécheresse consiste à identifier l'intervalle de temps pendant lequel les conditions hydriques sont déficitaires par rapport à des valeurs normales. Les seuils utilisés pour déterminer ces périodes dépendent largement des traitements statistiques et des normes utilisés, c'est pourquoi la définition d'une période de sécheresse peut fortement varier d'un pays à un autre.

Afin de déterminer une ou des périodes d'étude de sécheresse géotechnique, une étude simple dés données de température et de précipitation a été réalisée à partir des données de Météo-France sur la région Est parisienne (Fíg. 2). Elle est basée sur l'analyse de la pluviométrie efficace mensuelle corrigée et de l'estimation d'une courbe de tendance par moyenne mobile centrée (période de 11 mois) pour lisser les disparités pluviométriques mensuelles. La courbe de tendance fait ainsi apparaître les débuts et fins des périodes de déficit hydrique susceptibles d'intéresser l'étude du RGA par interférométrie radar.

La pluie efficace brute est calculée à partir de la formule simplifiée : $\mathrm{PE}=\mathrm{PP}-\mathrm{ETP}$, le bilan dans ce cas peut être négatif. On définit également la pluie efficace corrigée, PEc, pour laquelle les valeurs négatives sont remplacées par zéro. Les histogrammes donnés ci-dessus sont des valeurs en mm par mois.

Précipitation (PP) : le terme précipitation recouvre toutes les eaux météoriques, il concerne la pluie, la rosée, le brouillard, la neige, la grêle, le givre. L'unité employée est le millimètre d'eau par surface unitaire de $1 \mathrm{~m}^{2}$.
EVapotranspiration (ETP) : elle désigne toutes les pertes d'eau par retour direct à l'atmosphère sous forme de vapeur d'eau. L'évaporation étant le phénomène physique t la transpiration le phénomène biologique. Elle est qualifiée de potentielle puisque la valeur cálculée correspond à la timite maximale d'évapotranspiration d'une zone lorsque celle-ci ne subit aucune restriction en eau.

Pluie efficace (PE) : dans le cadre d'un bilan hydrologique, on définit la notion de pluie efficace qui correspond à la quantité d'eau infiltrée et ruisselée.

On peut noter les périodes relativement humides juste avant les sécheresses de 1989 et de 1996. De même, la période 2001-2002 est relativement abondante en précipitations. Une explication possible de la sécheresse géotechnique serait un fort contraste météorologique (tant au niveau des températures que de la pluviométríe) qui fait que l'état hydrique des sols se situe hors de son état d'équilibre. Cette étude simple fuxe deux périodes de sécheresse à étudier par interférométrie : la sécheresse de mars à juin 1996 et celle de 2003. Celle de 1989 ne peut être étudłée en raison de l'absence de données radar sur cette période.

\section{3}

\section{L'interférométrie différentielle}

\section{1}

\section{Principe}

Ĺinterférométrie radar désigne l'ensemble des méthodes "qui utilisent au moins deux images SAR complexes afin d'obtenir des informations supplémentaires sur les objets présents dans une seule image SAR, en exploitant linformation de phase du signal SAR n, (Bamłer et Hartl, 1998). Pour apporter de l'information, un paramètre d'acquisition doit être différent entre la première et la dewxième image. Cela peut être soit l'instant d'accuisition, soit la position et l'orientation du radar (interférométrie simultanée, par exemple) soit la fréquence ou la polarité de l'onde, émise ou reçue, ou encore le mode d'acquisition.

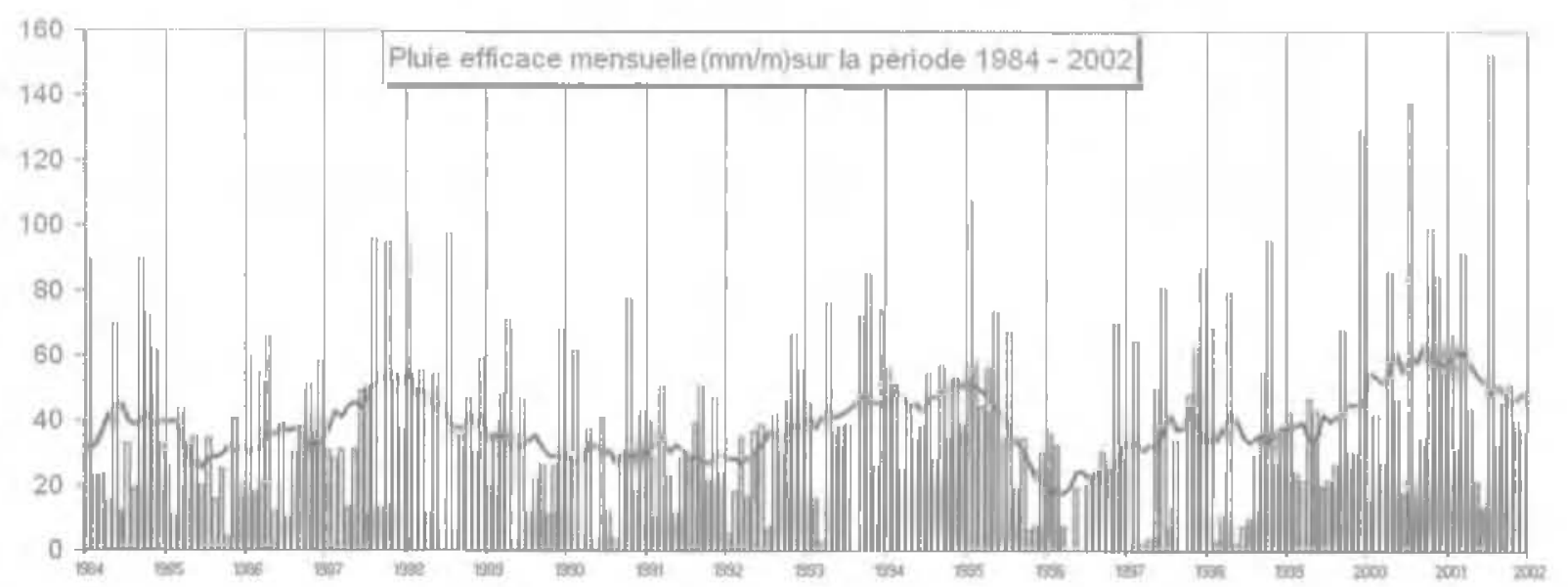

*6. 9 Histogramme de pluviométrie efficace corrigée (PEc) entre 1984 et 2002 sur I'Est parisien et courbe de tendance à partir de la moyente mobile calculée sur une période mobile de onze mois (Données : (Météo France) Fainfall Jistogram between 1984 and 2002 on the eastem Paris basin and the Moving average curve extracted with a period of 11 months (Data: ( Meteo France). 


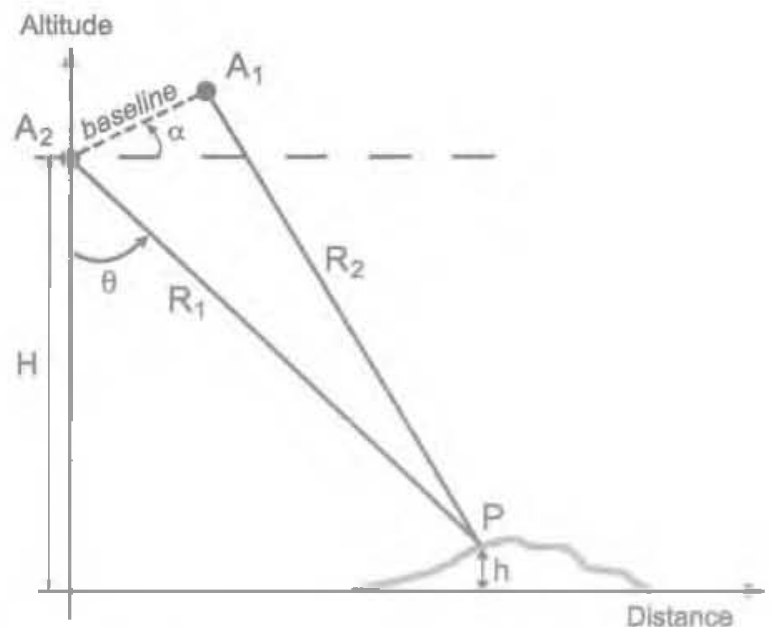

FI. 3

Géométrie d'acquisition en interférométrie. Les deux capteur's $\mathrm{A}_{1}$ et $\mathrm{A}$, sont séparés par une distance, la ligne de base. Idéalement. cette distance doit être la plus courte possible. Une cibie $\mathrm{P}$ est localisée dans la scène, à une hauteur $h . R_{1}$ et $R_{2}$ sont les distances respectives entre les capteurs et la cible. Geometry accuisition in interferometry.

The distance beween the two sensors $A_{4}$ and $A_{2}$ is called the baseline which must be as short as possible. The target $\mathrm{P}$ is at a distance $\mathrm{R}_{1}$ and $\mathrm{R}_{2}$ and an elevation $h$.

Pour l'étude des déplacements, le seul paramètre d'acquisition qui doit changer entre les deux images est la date d'acquisition entre les deux passages successifs. Dans ce type d'application, il est donc préférable cque la géométrie d"acquisition entre les passages successifs du capteur soit identique autant que possible. Dans un cas idéal où le satelilite repasse exactement au même endroit, on peut alors étudier la différence de phase entre les deux images (la répartition spatiale de ce déphasage et étudier ainsi l’origine de ce déphasage. Cependant, la relative maîtrise du positionnement des satellites ne permet pas d'avoir des géométries d'acquisition strictement identiques. La distance entre les deux satellites est appelée la ligne de base du couple (baseline en anglais) gui est l'un des paramètres de séjection des images radar pour produire des interférogrammes exploitables.

Les interférogrammes calculés font apparaitre des variations de phase sous forme de franges interférométriques colorées. Une différence de phase entre les deux images peut parfois se traduire par la présence de plusieurs franges. Chaque frange est équivalente à un déplacement dont l'amplitude est égale à une demiIongueur d'onde (2,8cm pour le capteur ERS1 et ERS2) selon la ligne de visée du capteur. Par déroulement des franges, on peut ainsi estimer une des composantes du vecteur de déplacement de la surface du sol.

La différence de phase (déphasage), résulte de plusieurs contributions décrites de la façon suivante :

$$
\Delta \phi=\Delta \phi_{\mathrm{d}}+\Delta \phi_{\mathrm{a}}+\Delta \phi_{\mathrm{l}}+\Delta \phi_{\mathrm{dc}}+\Delta \phi_{\mathrm{g}}+2 \mathrm{k} \pi
$$

$\Delta \phi$ : différence de phase ,

$\Delta \phi_{\mathrm{d}}$ : différence de phase liée au déplacement du sol, mesuré selon la ligne de visée;

$\Delta \phi_{\mathrm{a}}$ : différence de phase due au changement des conditions atmosphériques ;

$\Delta \phi_{1}$ : différence de phase liée à la topographie ;

$\Delta \phi_{\mathfrak{d c}}$ : différence de phase issue des décorrélations géometriques et temporelles :
$\Delta \phi_{\mathrm{r}}$ : différence de phase résiduelle (bruit) issue des traitements (recalage, enregistrement SAR).

L'analyse d'un interférogramme consiste principalement à distinguer les differentes contributions des phases au sein de l'image pour isoler la seule composante $\Delta \phi_{\text {d }}$ (mesure du déplacement). La précision de mesure pour cette méthode interférométrique est de l'ordre du demi centimètre (Fruneau et al., 2005), pourvu que les données spatiotemporelles de la technique (répétitivité de mesure égale à 35 jours, mesure dans l'axe de visée) soient adaptées au phénomène étudié.

\section{3.}

\section{Limites de l'interférométrie}

Linterférométrie DInSAR fonctionne si le décalage dans le temps n'est pas trop long (infërieur à dix ans), si la ligne de base est inférieure à un kilomètre, sì l'atmosphère est comparable au moment des deux accuisitions de donmées. Sous ces conditions, les deux images radar forment un couple interf́érométrique performant et permettent d'obtenir des franges interprétables en terme de déplacement.

\section{sti:}

\section{Perte de cohérence}

En pratique, l'interprétation des franges interférométriques est rendue difficile par la perte de cohérence temporelle et/ou spatiale (ou géométrique) entre les deux acquisitions. La cohérence est une mesure du degré de corrélation de la phase entre les deux images radar prises sur une même zone avec un certain intervalle de temps. Plus la cohérence est élevée, plus l'interférogramme est lisible. Par exemple, la présence de végétation diminue ou annule la cohérence. A l'ínverse, un milieu densément urbanisé favorise la concentration de réflecteurs RADAR et améliore la lisibilité de l'interférogramme. Cette cohérence peut être diminuée par (1) la décorrélation spatiale et (2) la décorrélation temporelle.

(1) La décorrélation temporelle est liée aux changements physiques de la surface entre les deux observations. Cela peut servir d'indicateur de l'état de la surface du sol et ses modifications entre les deux acquisitions. Une illustration de cette décorrélation est proposée à la figure 4 : on peut observer que sur un interférogramme avec une faible période (ich un mois) entre les deux acquisitions sera plus cohérent, notamment aı riveau des champs d'agriculture, qu'un inter férogramme avec une période entre images radar de plusieurs années (3 ans pour le second couple).

(2) La décorrélation géométrique résulte des variations dans la géométrie d'acquisition des images. Dès lors que la ligne de base perpendiculaire entre les deux positions du radar aux deux dates d'acquisitions des images est non nulle, il existe une différence d'angle d'incidence qui fait que la mesure ne se répète pas exactement au même endroit. Cette décorrélation spatiale, est d'autant plus importante que lá ligne de base perpendiculaire est élevée. Certains couples d'images, pour lesquelles la ligne de base dépasse la ligne de base critique, ne donneront pas des interférogrammes exploitables. 
De même, ce type de décorrélation spatiale peut résulter de variations de l'angle de dépointage du radar, qui ne vise pas perpendiculairement à sa trajectoire, mais vers l'avant ou l'arrière. Lne défaillance des Gyroscopes qui contrôlent l'altitude d'ERS-2, à partir de février 2000 est à l'origine de fortes variations de cet angle de dépointage et donc du Doppler des images. Seuls les couples dont la différence de Doppler est inférieure à 400 Hz sont donc calculés.

\section{axio}

\section{Les artefacts atmosphériques}

Les artefacts atmosphériques viennent également perturber l'interprétation des interférogrammes et doivent donc être décrits. Les hétérogénéités a tmosphériques locales (présence de nuages, pluie...) introduisent des franges atmosphériques et des anomalies de formes variables (globulaire, effilếe ...) sur les interférogrammes et qui ne peuvent pas être facilement séparées des franges de déolacement. Une illustration à la Figure 5a montre les effets sur l'interférogramme de ce type d'artefact.

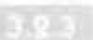

\section{Les artefacts liés aux traitements}

Erfín d'autres effets indésirables peuvent apparaître au sein des interférogrammes comme l'apparition d'une frange liée à une mauvaise connaissance de la géométrie orbitale lors de l'acquistition des images (voir Fỉgure 5b). Il apparaît ainsi des franges résiduelles au sein des interférogrammes qu'il est possible de corriger.

\section{3}

\section{Critères de sélection des couples interférométriques}

Trois critères de sélection interviennent dans le choix des images radar pour calculer un interférogramme de qualité. Ce sont les périodes de sécheresse, la ligne de base et la valeur du doppler. Selon que ces critères sont plus ou moins respectés, on peut juger de l'intérêt d'un interférogramme avant son calcul.

Les périodes de sécheresse sont un critère important de sélection dans ce type d'étude. Il motive le choix des couples et justifie de recalculer des interférogrammes même si les autres paramètres sont en limite de qualité. En effet, chaque interfërogramme calculé implique deux images. Le fait gue l’une ou les deux images appartiennent à ces périodes va déterminer l'interprétation qui en sera faite. En effet, certains mouvements sont attendus selon que l'interférogramme couvre ou non la période sensible de sécheresse. En dehors de ces périodes de sécheresse, il sera intéressant de suivre sur le long terme les effets consécutifs au tassement, fusqu'à deux ans après l'épisode climatique déclencheur. Enfin, i] sera également intéressant d'observer le retour à l'équilibre des sols sur les interférogrammes.
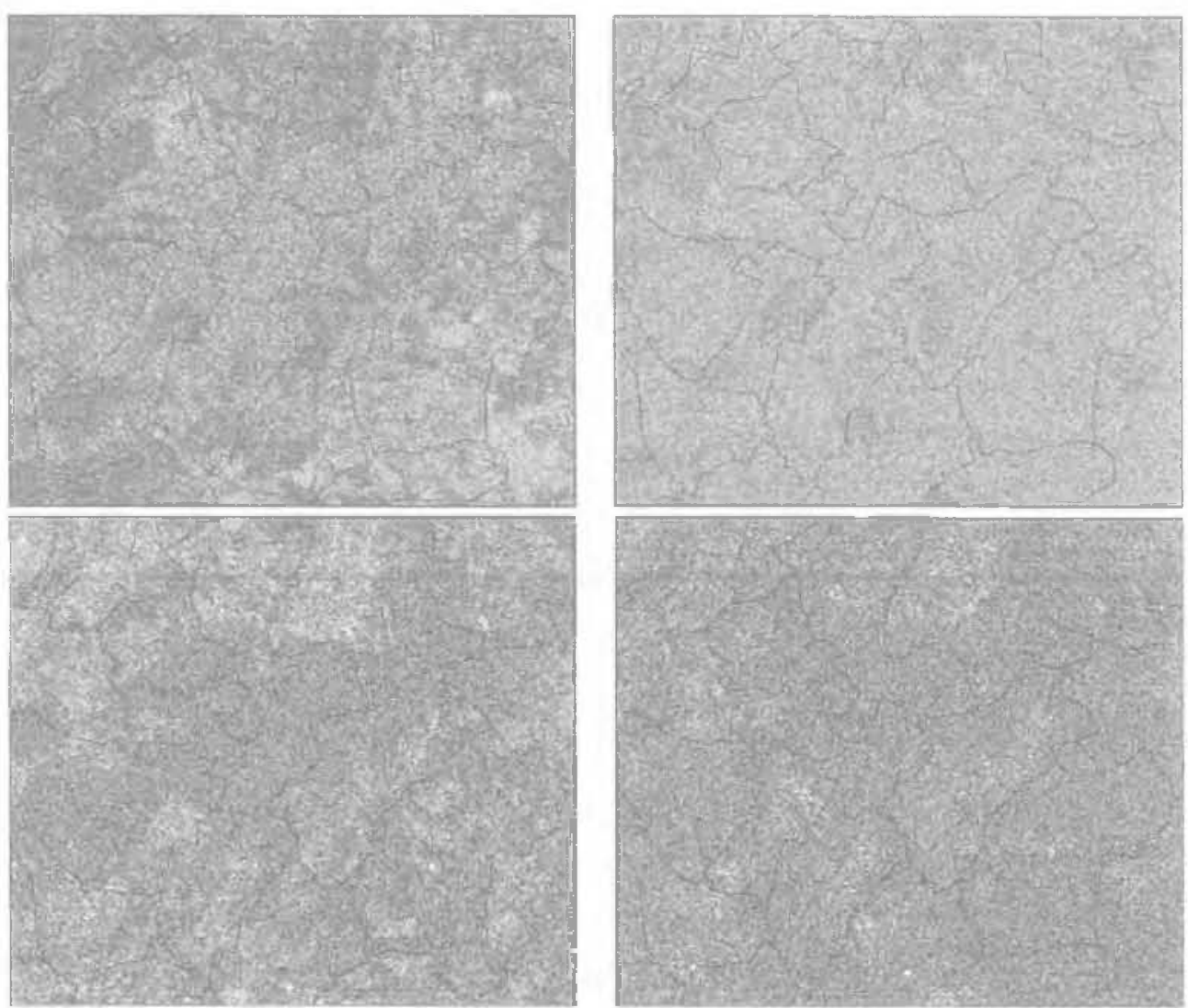

FG. I Ilustration de la perte de cohérence temporelle. Example of the temporal coherence loss. Figures disponibles en couleur sur https//www:univ-mlv.fr/g2i/kaveh/ 

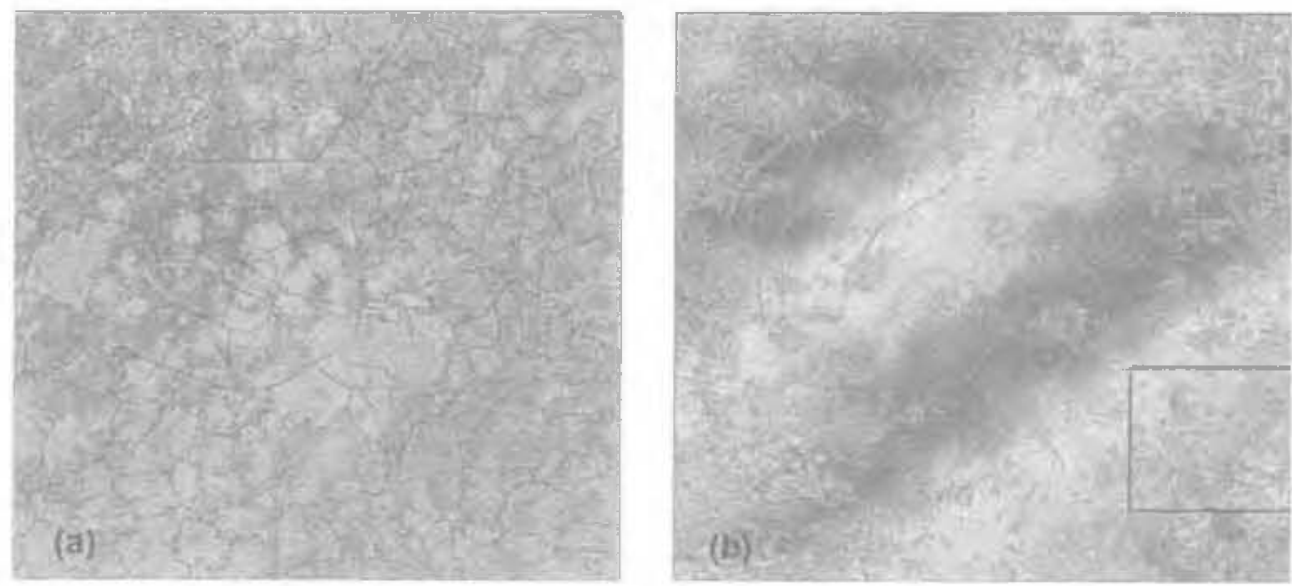

a) Exemple d'un interférogramme affecté par des artefacts atmosphériques :

b) Exemple d'un interférogramme avec deux franges orbitales.

a) Example of an interferogram affected by amospheric artefacts; b) Exanple of an interferogram characterized by two orbital fringes.

Figures disponibles en couleur sur http:/www,uriv-mlvfr/g2i/kaveh/

La ligne de base ou " baseline est un paramètre essentiel dans la sélection des données radar pour constituer des couples interférométriques. C'est une variable qui indique la qualité des interférogrammes produits. Une sélection avec une ligne de base de 200 mètres a été ici réalisée, même si dans la pratique, les interférogrammes facilement interprétables ont une valeur de ligne de base inférieure à 100 mëtres.

Enfin, un paramètre lié aux traitements des données SAR, la valeur du doppler centroide, a été pris en compte dans la sélection des images radar. En têllédétection radar, la précision du doppler centroïde affecte directement la qualité des ímages radar. Sá connaissance est donc importante pour l'application interférométrique envisagée. En effet, les échos qui portent l'information de phase et d'amplitude sont enregistrés par le capteur radar en utilisant un effet doppler, qui est utilisé en traitement du signal pour reconstruire les images. Or, suite à une défaillance du satellite ERS-2 en février 2000, les valeurs du doppler centroide ont des variations importantes, ce qui réduit le nombre d'interférogrammes à calculer. Ainsi, la sélection des images radar pour constituer un interférogramme exploitable est faite de telle sorte que la différence entre les doppler des deux images n'excède pas $400 \mathrm{~Hz}$ en valeur absolue.

Donc, même après une sélection par la ligne de base et les dates, tous les interférogrammes ne sont pas exploitables. Avec les données des missions ERS-1 et ERS-2 en mode ascendant et descendant et en appliquant les critères de sélection exposés plus haut, plus de 300 interférogrammes ont été calculés, mais principalement sur la période 1995 à 2000. Ces interférogrammes présentent des qualités variables, seuls certains étant réelkement exploitables pour l'application envisagée.

\section{Application de l'interférométrie à l'Est parisien}

Cette parțe décrit les résultats obtenus par l'interférométrie DInSAR appliquée à l'Est parisien pendant les périodes de sécheresse. En premier lieu une description de la zone d'étude est présentée, puis des planches illustrées d'interférogrammes et de documents thêmatiques sont décrits et analysés.

\section{1}

\section{La zone d'étude}

Une zone d'intérêt a été définie et deux sites ont été sélectionnés dans lEst parisien. Après avoir précisé leur emprise géographique, une analyse géomorphologique et géologique est présentée, ainsi que l'analyse de la sinistralité effectuée pour caractériser l'ampleur spatiale du phénomène. L'ensemble de ces informations est essentiel pour la bonne réalisation des travaux en interférométrie radar.

\subsection{5}

\section{Localisation et occupation du sol}

Un site a été sélectionné sur des zones urbaines concentrant de nombreux dommages sur les bâtiments (Fig. 6) entre les départements du Val-de-Marne (94) et de Seine-Saint-Denis (93) s'étendant en partie sur huit communes de l'Est parisien. Les rapports du BRGM recensent plusieurs centaines de sinistres dans ce département. Les communes couvertes par le site d'étude (Bry-sur-Mame, Champigny-sur-Marne, Fontenay-sous-Bois, Nogent-sur-Marne, Le Perreux-surMarne) sont renseignées dans la base de données avec les documents thématiques. La zone est centrée sur la commune de Nogent-sur-Marne, avec une emprise qui a été choisie de telle sorte que le rendu cartographique des figures soit à l'échelle du $1 / 50000$.

Le fichier du mode d'occupation du sol a MOS 2003 n de l'Institut d'Aménagement et d'Urbanisme de la région lle-de-France (IAURIF) à 21 postes - issu de la photo-interprétation d'imageries aériennes -, décrit l'occupation du sol, avec entres autres des informations sur le caractère individuel ou collectif des bâtiments. Disponible pour l'Est parisien, cette classification permet de calculer la surface occupée par les principaux types de bâtis édifiés pour la zone d'étude. La répartition des classes par ordre d'importance décroissante est donnée par le tableau suivant. 


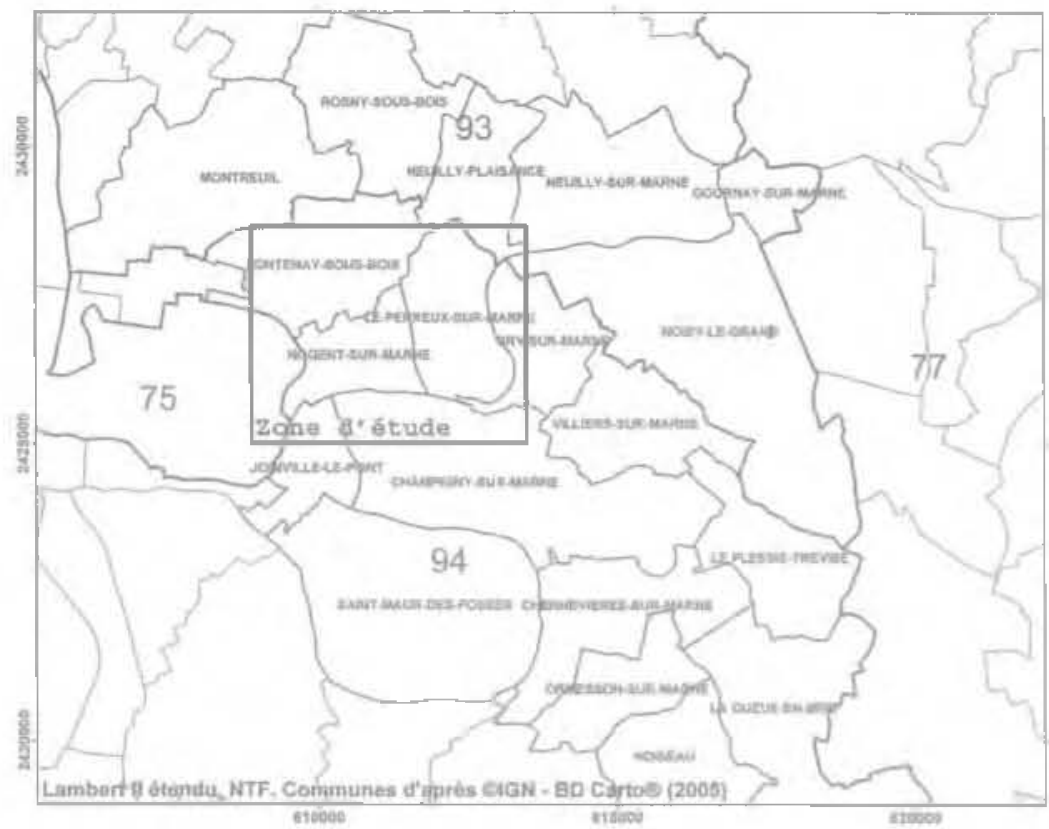

F0. Localisation de la zone d'intérêt au seìn du département du Val-de-Marne (94) ; limite départementale en gras, limite des commumes en trait fin.

Location of the test site in the Val-de-Marne district. District boundary in heavy black line, cities boundary in light black bine.

thateur Statistique d'occupation du sol sur' les zones d'études à 21 postes, classés par ordre d'importance de la superficie (Source : MOS IAURIF)

Surface statistic of 21 land cover classes ranked in sufface order of inportance.

\begin{tabular}{|c|c|c|c|}
\hline Code & Libellé & Surf. $\left(\mathrm{km}^{3}\right)$ & $\%$ \\
\hline 9 & Habitat individuel & 13,9 & 42,7 \\
\hline 10 & Habitat collectif & 4,3 & 13,2 \\
\hline 6 & Parcs et jardins & 3,1 & 9,5 \\
\hline 20 & Transports & 2,6 & 8,0 \\
\hline 12 & Activités secondaires & 1,8 & 5,5 \\
\hline 1 & Bois & 1,4 & 4.4 \\
\hline 7 & Sport (espaces ouverts) & 0,9 & 2,9 \\
\hline 15 & Équipement d'enseignement & 0.8 & 2,4 \\
\hline 8 & Terrain vacant & 0,7 & 2,1 \\
\hline 4 & Eald & 0,7 & 2,1 \\
\hline 18 & Locaux, administration. & 0,5 & 1,5 \\
\hline 13 & Activités tertialres & 0,4 & 1,4 \\
\hline 5 & Autre rural & 0,4 & 1,4 \\
\hline 17 & Cimetière & 0,2 & 0,7 \\
\hline 21 & Chantiers et divers & 0.2 & 0,5 \\
\hline 14 & Sport [constut] & 0,1 & 0,4 \\
\hline 2 & Grandes cultures & 0.1 & 0,4 \\
\hline$\pm \delta$ & Équipement de santé & 0,1 & 0,3 \\
\hline 11 & Habitat aute & 0.1 & 0,2 \\
\hline 19 & Grands equipennents & 0,0 & 0,1 \\
\hline \multirow[t]{2}{*}{3} & Autres cultures & 0,0 & 0,1 \\
\hline & TOTAL & 32,6 & 100,0 \\
\hline
\end{tabular}

Pour une surface totale de $32,6 \mathrm{~km}^{2}$, les bâtiments résidentiels représentent $55,5 \%$ de la surface urbanisée et c'est l'habitat individuel gui arrive en première position avec près de $42.7 \%$ d'occupation du sol urbanisé, suivi de très loin par l'habitat collectif $(13,2 \%)$. On constate également l'importance des espaces arborés publics qui représentent $16 \%$ de la surface de la zone d'étude (parcs, forêts et plans d'eau). Ensuite viennent les transports eł les activités secondaires avec respectivement $8,0 \%$ et $5,5 \%$.

Ces chiffres traduisent d'une part le caractère majoritairement résidentiel du mode d'habitat dans les zones d'étude choisies et d'autre part le caractère individuel des bâtiments. Ce tissu urbain est caractéristique d'une zone périurbaine secondaire, avec de nombreux pavillons accompagnés de jardins privés.

\section{sitas \\ Slnistralité}

Une étude préalable de la sinistralité a été réalisée afin de développer des bypothèses sur les mouvements de la surface du sol lié au RGA. Pour cette courte analyse des désordres provoqués par le RGA, il est important d'évaluer l'évolution temporelle et l'extension spatiaje du phénomène.

Les déclarations des sinistres sur les biens privés auprès des assureurs et des collectivités ainsi que les constats d'experts permettent de dresser un premier bilan du phënomène. Ces données ont été rendues publiques par le BRGM pour un département voisin. la Seine-et-Marne (Donsimoni et al., 2006). Néanmoins, notons que les déclarations de sinistres peuvent intervenir plusieurs mois après la date effective du début du sinistre. Cela esł en partie lié à une observation tardive ou à une déclaration différée. On notera qu'une à deux années peuvent s'écouler avant qu'une commune soit déclarée en état de catastrophe naturelle par l'État.

On observe généralement que les dommages se répercutent à long terme après un épisode de sécheresse (Figure 7). L'étude des dates de sinistralité monlre que la sécheresse de 1989 précède des sinistres déclarés en 1991 et 1992 en saison humide. Le nombre de sinistres déclarés en 1989 est à peu près équivalent au nombre de sinistres survenus en 19y1 et 19g2. Il faut attendre l'année 2003 pour observer à nouveau une 
sinistralité comparable en nombre dé dêclarations à celle de 1989.

Cette etude montre que le RGA provoque des sinis tres sur une période de plusieurs années, longtemps après l'épisode de sécheresse. La sécheresse, qui est un événement ponctuel à l'échelle d'une saison se tràduit par un risque RGA sur plusieurs années en fonction de la susceptibilité du sol au retrait. La sécheresse provogue donc un retrait, qui est le moteur de la déformation. Cette phase initiale du RGA est suivie d"un gonflement qui intervient de manière inégale tant dans le temps que dans l'espace. On observe aussi sur la figure 7 que la sécheresse de 1996/1997 n'a pas apporté autant de déclarations de sinistres liés au RGA qu'en 1989 dans le département de la Seine-et-Marne (77).

Cependant, dans la mesure où les données radar d'archives ERS ne permettent qu'une étude à partir de 1993, seułs la sécheresse de 1996/97 et l'épisode caniculaire de 2003 seront donc étudiés.

\section{$4 \sin ^{4}$}

\section{Éléments de géologie/géomorphologie}

Le relief de la zone d'intérêt est constitué de trois principales unités : Ia plaine alluviale de la Marne, le plateau et ses versants (Fig. 9). Les flancs des plateaux présentent des pentes prononcées recoupant les formations géologiques subhorizontales. Au niveau des talwegs, les pentes sont plus douces et les formations sous-jacentes aux limons de plateau affleurent davantage. L'altitude de la plaine alluviale n'excède pas les 35 mètres. La plaine alluviale est composée d'alluvions quaternaires : sables et graviers recouverts par des limons d'inondation. Sur les flancs des versants affleurent les couches de la série éocène et oligocène avec de la base au sommet : calcaire de Champigny ou masses et marnes de gypse, marnes supra-gypseuse, argile verte de Romainville et glaise à Cyrènes, caicaire de Brie et argiles è meulières. Au sommet des plateaux, une cotiverture de limon des plateaux est présente (Fig. 8a).

Parmi ces formations citées pius haut, le calcaire de Brie $(g, b)$ par sa nature calcaire est peu sensible au phénomène RGA, bien qu'il puisse contenir à la base quelques niveaux mamo-calcaires, voire argileux, et bien qu'il puisse présenter localement des poches de décalcification remplies de matériaux argłleux. De même, les limons des plateaux (LP) sont peu sensibles au retrait/gonflement, car principalement constitués de matériaux limoneux, même si localement la distinction entre limons de plateaux et argiles à meulières sous-jacentes est délicate. Les autres formations géoIogiques, par leur nature mameuse ou argileuse, sont

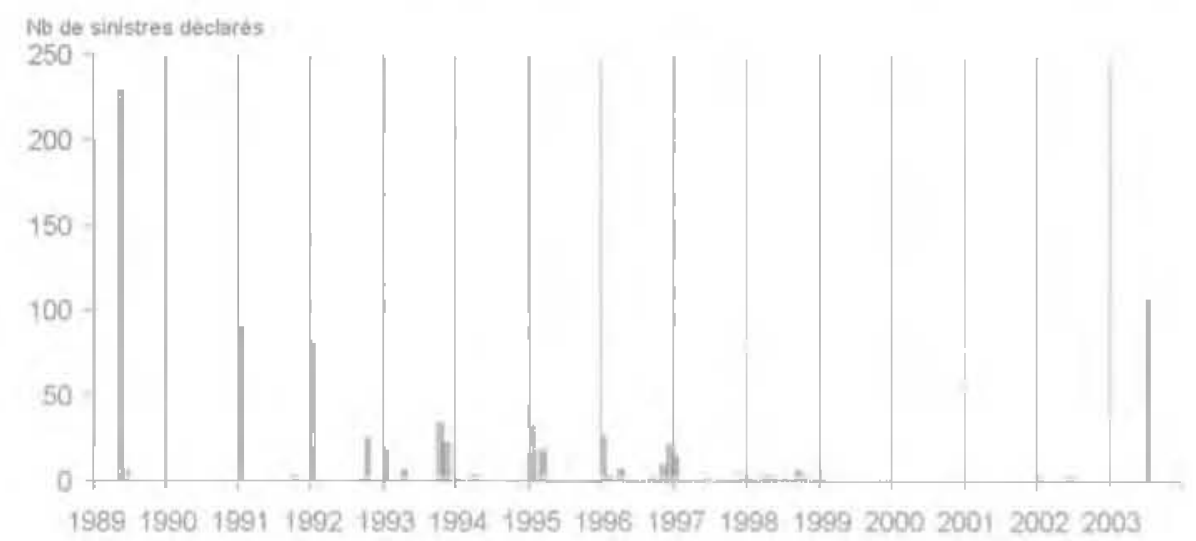

HG. 7 Dates des déclarations de sinistres de bảtiments pour le département de Seine-et-Marne (77) entre 1989 et 2004 (données extraites de Donsimoni et al. 2006).

Date of the damaged houses declaration for the Seine-et-Mame District (77) between 1909 and 2004 (data coming from Donsinoni ef al, 2006 ).
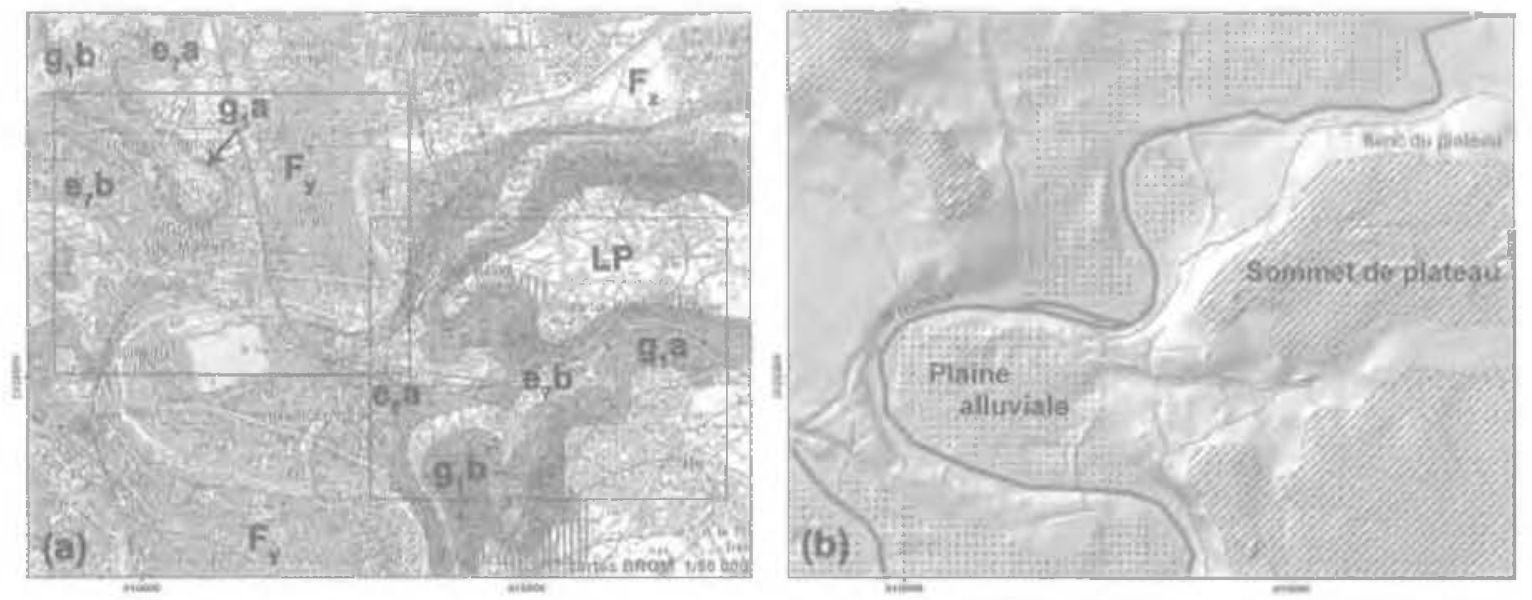

FG. 8 a) Extrait légendé de ta cartographie géologique du BRGM (Soyer et al., 1958) ; b) Descriptif géomorphologique simplifié sur le zone d'intérêt.

a) Detail of the BRGM mapping (Soyer at al.. 1958 ); b) Simplified geomorphologic description of the test site. 
potentiellement sujettes au retrait/gonflement. Il s'agit d'étudier le lien entre la sinistralité et l'extension des formations géologiques décrites cartographiquement par le BRGM

$\mathrm{e}_{7} \mathrm{a}$ : calcaire de Champigny et marnes à pholadomies indifférenciées (Eocène supérieur, Priabonien, Ludien inf. à Ludien moy., marnes ludiennes [faciès de transition), et masses et marnes du gypse (Éocène supérieur, Priabonien, Ludien moyen) :

e,b : marnes supragypseuses (Éocène supérieur, Priabonien, Ludien supérieur) ; les mames bleues d'Argenteuil et les marnes blanches de Pantin;

$\mathrm{G}_{\mathrm{f}} \mathrm{a}$ : argile verte de Romainville et glaises à cyrènes (Oligocène, Rupélien, Sannoisien inférieur);

$g, b$ : calcaire de Brje et argiles à meulières (oligocène, Rupélien, Sannoisien):

LP : limons des plateaux :

$F_{y}$ : aliuvions anciennes;

$F_{z}$ : alluvions modernes.

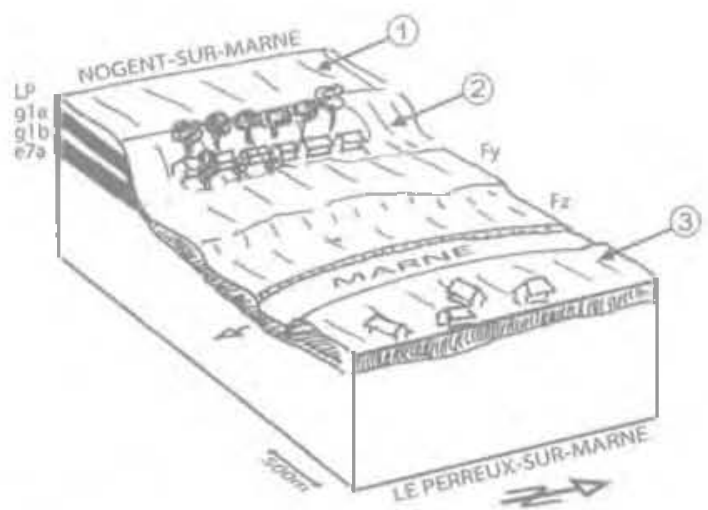

F16. 9 Représentation 3D simplifiée de la zone d'étude de Nogent-sur-Marme.

1- Plateaux, soumis au RGA et retrat des nappes temporaires, 2- Flanc de coteawx. sensible au fluage des sols et au HGA, 3Zone alluvionnaire humide soumise à l'aléa sécheresse par la baisse hydraulique de la nappe sous jacente.

3D view of the Nogent-Sur-Marme area.

1- Plateau submitted to the drought, 2- Flank Sensitive to the soil creep and drought, 3-AHuvial plain submitted to topographic movement due to the seasonal piezometric variation.

\section{9.}

\section{Analyses}

Les planches en couleurs qui montrent les résul tats des interférogrammes sont placées à la fin de l'article en noir et blanc et sont disponibles en couleur sur http:/Www.univ-mivifr/g2i/kaveh/

La planche 1 présente une série de vignettes focalisées sur les villes de Nogent-sur-Marne et du Perreux-sur-Marne. Deux agrandissements $A_{1}$ et $A_{2}$ sont extraits de l'orthophotographie A. Les maisons qui ont été déclarées sinistrées pour le RGA entre 1989 et 2002 (étoiles noires) sur la commune de Nogent-sur-Marne sont notamment alignées N/S perpendiculairement à la Marne sur une zone de forte pente (planche $1, \mathrm{~A}_{1}$ ). Elles sont situées sur des roches argileuses gonflantes (g.a et $\mathrm{g}_{1} \mathrm{~b}$ ) d'âge éocène à oligocène supérieur (planche 1 , C). Ces maisons individuelles sont encadrées par deux espaces arborés (planche 1, A et A) . La combinaison des trois facteurs 'végétation arborée', 'forte pente', et 'sol gonflant argileux', est certainement à l'origine de cette forte sinistralité. Cette zone appartient bien à un aléa fort d'après la cartographie du BRGM (planche 1, B). L'interférogramme brut (planche 1, E) et l'interférogramme masqué (planche $1, F$ ) à une cohérence de 0,6 soulignent et révèlent une forte cohêrence sur les maisons et bâtiments et, à l'inverse, une faible cohérence sur les zones boisées. La présence de pixels contigus de différentes couleurs sur les interférogrammes souligne la disparité des amplitudes de déplacements des bẩiments, mais cela reste difficilement exploitable du fait du bruit important

La seconde illustration (planche 1, A, ) est située sur la ville du Perreux-sur-Marne, dans la plaine alluviale de la Marne où se sont déposées les alluvions holocènes $F_{y}$ et les alluvions récentes $F_{z}$ (planche $1, C$ ). Ces alluvions argileuses, qui ont des propriétés gonflantes, correspondent à des dépôts de plaine d'inondation. L'agrandissement de l'orthophotographie (planche 1, A.) montre l'importance de l'habitat pavillonnaire et la présence d'arbres isolés. La sinistralité est caractérisée par des aléas faibles à moyens (planche 1, D). Linformation délivée par les interférogrammes (planche 1. $E$ et $F$ ) révèle une faible cohérence liée à l'occupation pavillonnaire jardinée, rendant les interférogrammes difficilement exploitables. La sinistralité est très dispersée et difficile à interpréter.

La planche 2 présente une série d'interférogrammes calculés à partir de couples d'images radar indépendantes sur la zone d'étude. Uñ rappel de la topographie (planche 2A) et de la géologie (planche 2B) est présenté en haut de la planche afin d'apprécier te contexte général.

Ces six interférogrammes révèlent des déformations soulignées par des flèches noires. Les numéros d'orbite et les dates d'accquisition des couples sont indiqués respectivement en bas à gauche et en bas à droite des vignettes. Les cinq premiers extraits d'interférogrammes révèlent un déphasage du signal radar au niveau de la transition entre la formation $F$, et la formation $e_{7} b$. On peut observer la proximité de ce déphasage avec les sinistres localisés juste en amont, ainsi qu'avec une rupture de pente qui caractérise le changement lithologique entre les alluvions $\mathrm{F}_{y}$ et les mames de l'Éocène supérieur e $e_{7} b$. Ce déphasage est croissant (rapprochement du sol vers le capteur) entre les années 1996 et 1999 pour les quatre premières vignettes, et seul l'interférogramme 15155022 entre le 04.08 .95 et le 05.04.96 présente un déphasage négatif (éloignement du sol dans la direction de visée du capteur radar). On montre ainsi un retrait à peine perceptible pendant la fin de l'année 1995 et avril 1996 de l'ordre de 0,3 cm et un gonflement des sols de $0,4 \mathrm{~cm}$ jusquíen 1998 dans le sens de visée du satelijte. Une discussion sur l'amphtude de ces valeurs est effectuée ci-après. La dernière vignette représentée par le couple 24695/17046 pour la période entre du 04,04.1996 au 24/071998, montre un déphasage positif interprété comme un gonflement sur la rive Est du Perreux-sur-Marne. $\square$ coïncide également avec des sinistres sur une zone alluvionnaire. On note des signaux de déphasage positif également sur les berges de la Marne. 


\section{Discussion}

Sur le terrain, les déplacements engendrés par le RGA affectent une zone comprise entre la dizaine de mètres carrés et plusieurs centaines de mètres carrés en planimétrie pour des mouvements verticaux de l'ordre au maximum de quinze centimètres. Cependant, les mouvements détectés par interférométrie sont bien inférieurs, car de l'ordre du demi-centinètre sur les surfaces urbaines de Nogent-sur-Marne. Dans la mesure où la technique interférométrique DInSAR décrit principalement les mouvements liés aux réflecteurs des bâtiments, on parvient à expliquer cet écart. En effet, on mesure par interférométrie la réponse du bâtiment aux sollicitations des mouvements induits par le RGA.

Deux types de mouvement sant à distinguer : (1) celui des bêtiments en général, mesuré par interférométrie radar avec une amplitude de quelques millimètres et une surface de plusieurs dizaines de $\mathrm{m}^{2}$, et (2) les mouvements du sol caractérisés par une amplitude pluricentimétrique. Hanssen, (2001) définit le champ d'application de l'interférométrie différentielle en prenant en compte l'amplitude et l'extension spatiale des mouvements étudiés (Fig. 10). Cette Ëgure a été modifiẻe en ajoutant un bloc \& RGA * avec les dimensions spatiales de ce type de mouvement observé. Ainsi, les déformations de la surface du sol observées et liées au RGA sont situées à la limite supérieure, du champ d'application de la méthode DInSAR (Fig. 10, bloc RGA. coin haut gauche), tandis que les mouvements détectés sur le bâti (amplitude millimétrique et forte extension) se placent dans le champ d'application de l'interférométrie (Fig. 10, bloc RGA coin en bas à droite).

Les calculs réalisés avec la technique interférométrique DinSAR appliquée à la sécheresse 1996/97 montrent donc des límites. La rêponse des bâtiments aux déformations engendrées par le RGA n'est pas homogène dans le temps et dans l'espace. Quelques signaux détectés ont pu être mis en relation avec la sinistralité mais cela nécessite une confirmation terrain. Cependant, les déformations discrètes ä l'échelle de petits bâtinents sont rarement décrites.

Parmi les facteurs environnants, participant au phénomène RGA, les surfaces végétalisées et arborées, caractérisées par leur forte évapotranspiration, sont souvent la source des nombreux désordres constatés. Or, ces zones ont une très faible cohérence en interfé rométrie radar, et sont donc peu identifiables au sein des interférogrammes. La topographie, en partículiter les pentes, est un facteur de prédisposition important, car il joue sur la stabilité du terrain pendant le cycle de dessiccation/hydratation du sol. A une échelle régionale, f'étude géomorphologique du paysage permet de mettre en évidence les zones à risque. A une échelle Iocale, des études de pentes précises sont nécessaires. Enfin, la nature du sol décrite par la carte géologique est certainement un point de départ dans l'étude de la susceptibilité au RGA. La méthodologie du BRGM est confirmée la sinistralité étant globalement liée à l'aléa cartographié.

Lanalyse des facteurs relatifs à la topographie, à la végétation et au type de sol révèle que la description du tissu urbain et de son organisation est nécessaire. Le mode d'occupation du sol de l'LAURIF, permet de situer les zones urbanisées, c'est-à-dire l'enjeu et plus particu- lièrement les zones urbaines secondaires pavillonnaires. Les informations ainsi extraites, telles que la proximité d'espace arboré, la pente du terrain ou la présence d'un terrain sensible au RGA, sont traitées sous un systeme d'Information Géographique pour produire une cartographie du risque et des dommages liés au RGA.

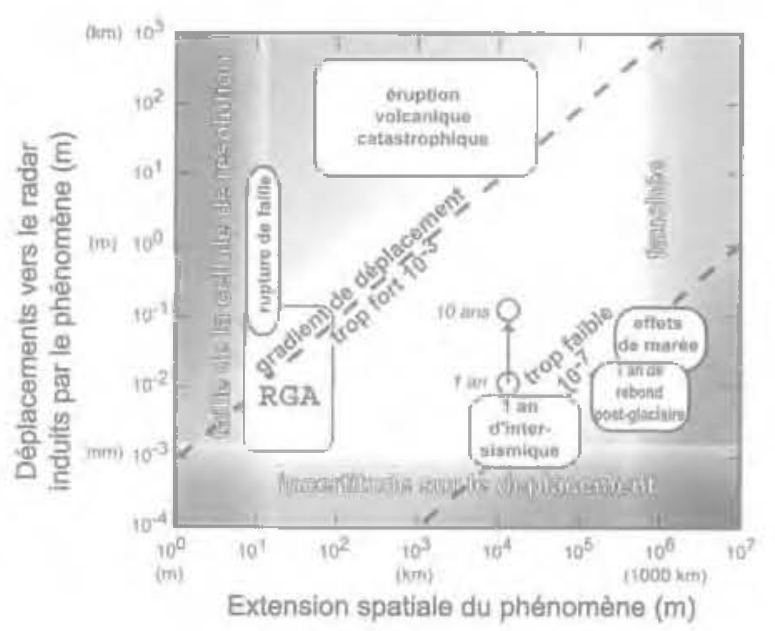

FG. 10 Domaine d'application de l'interférométrie radar (Massonnet et Feigl, 1998; Harssen, 2001 ; Pathier, 2005, modifiè).

Radar interferometric application domain (modiffed from Massomnet et Feigl, 1998; Hanssen, 2001: Pethier, 2005).

\section{5}

\section{Conclusion}

Cette approche multisource et pluridisciplinaire permet de mieux comprendre les déplacements de la surface topographique dans les zones soumises au retrait/gonflement. Un Système d'Information Géographique (SIG) est utilisé ici afin de mieux comprendre les origines de la sinistralité liée au retrajt/gonflement des sols argileux. Les résultats d'une cartographie optimisée du RGA à l'aide de la combinaison de données issues de la télédétection intégrée dans un SIG pourraient apporter des éléments concrets utiles aux gestionnaires et assureurs.

Plusieurs exemples sont issus de IFEst de l'Île-deFrance, région fortement affectée par la sécheresse géotechniqué. On constate que la proximité de zones fortement arborées, à forte pente et de formations géologiques argileuses gonflantes d'âge oligo-éocène sont autant de facteurs de prédisposition. L'interférométrie radar (DInSAR) est testée ici dans un but de localisation, de caractérisation et de quantification des amplitudes des déformations liées au RGA. Elle souligne des déplacements qui ne sont pas toujours facile à interpréter. En effet, les résultats observés sont limités du fait : (1) de la faible cohérence de la zone d'étude liée pro parte à la nature du tissu perrurbain thabitat individuel, pavillonnaire disparate, jardiné et arboré) : (2) du faible nombre d'images radar ERS et Envisat utilisées et actuellement disponibles sur les périodes de sècheresse et de déficit hygrométrique; (3) de la non-continuité des déplacements engendrés par le RGA dans le temps ; et, anfin (4) de la forte évolution 

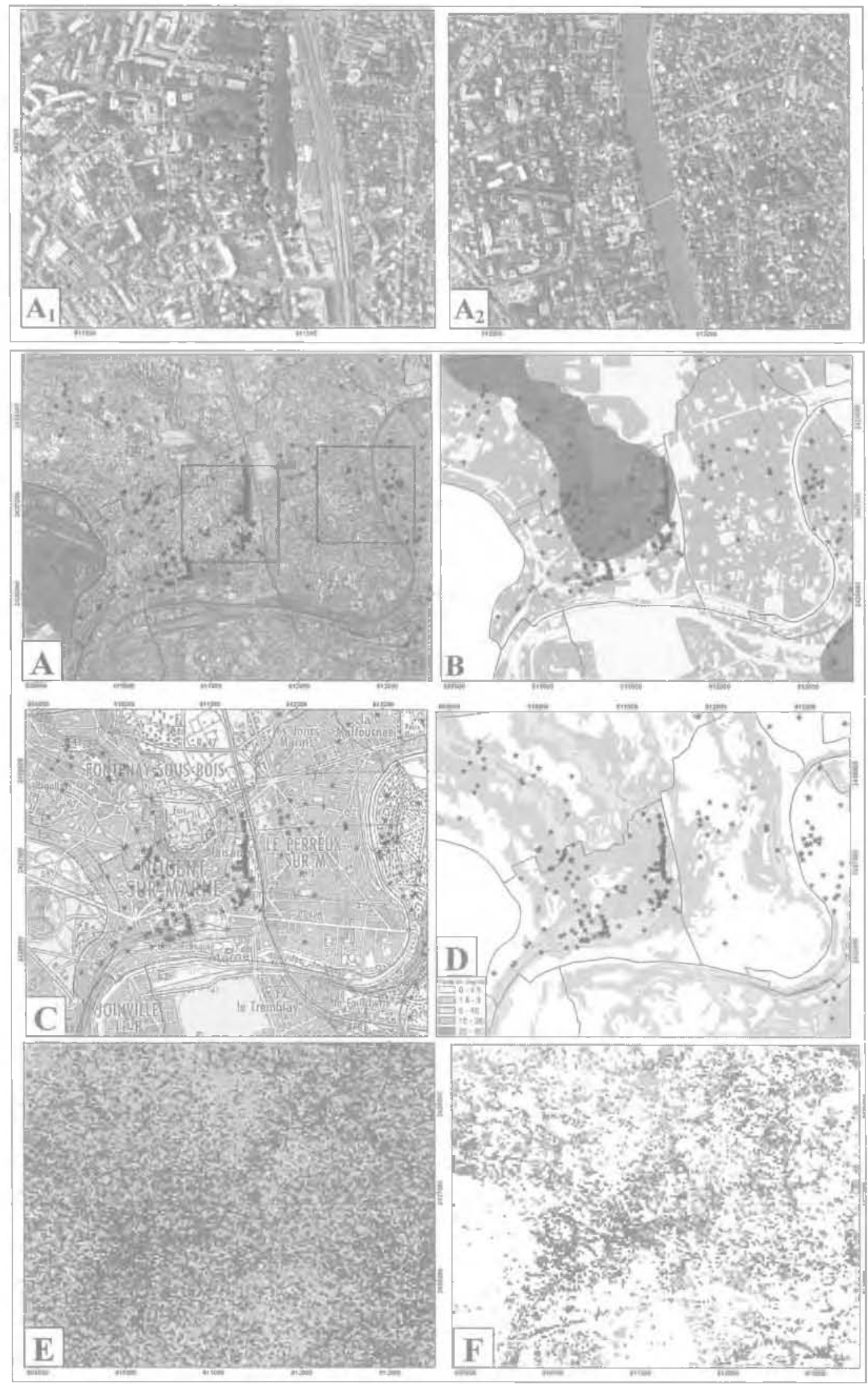

Planke 1 Zone de Nogent-sur-MarTe.

A, A1, A2- Orthophotographies; B- Carte d'aléa BRGM, modiffé avec le bâti (MOS LAURIF) ; C- Carte géologique de la figure 8a; D-Carte de pente ; E, F- Interférogramme 7334_20360 avec et sans masque de cohérence de 0,6 (disponible sur http:/www.univ-mlv.fr/g2i/kaveh/)

Nogent-sur-Marne area.

A A1, A2- Aerials photos; B- Bisk map issted from BRGM, modified, and anthropic builing area MOS IAURLF]; CGeological map fig..$^{\circ}$; D- Slope map; E. F- Interferogram 7334_20360 with and without a coherence mask of 0.6 (avaible on

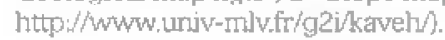



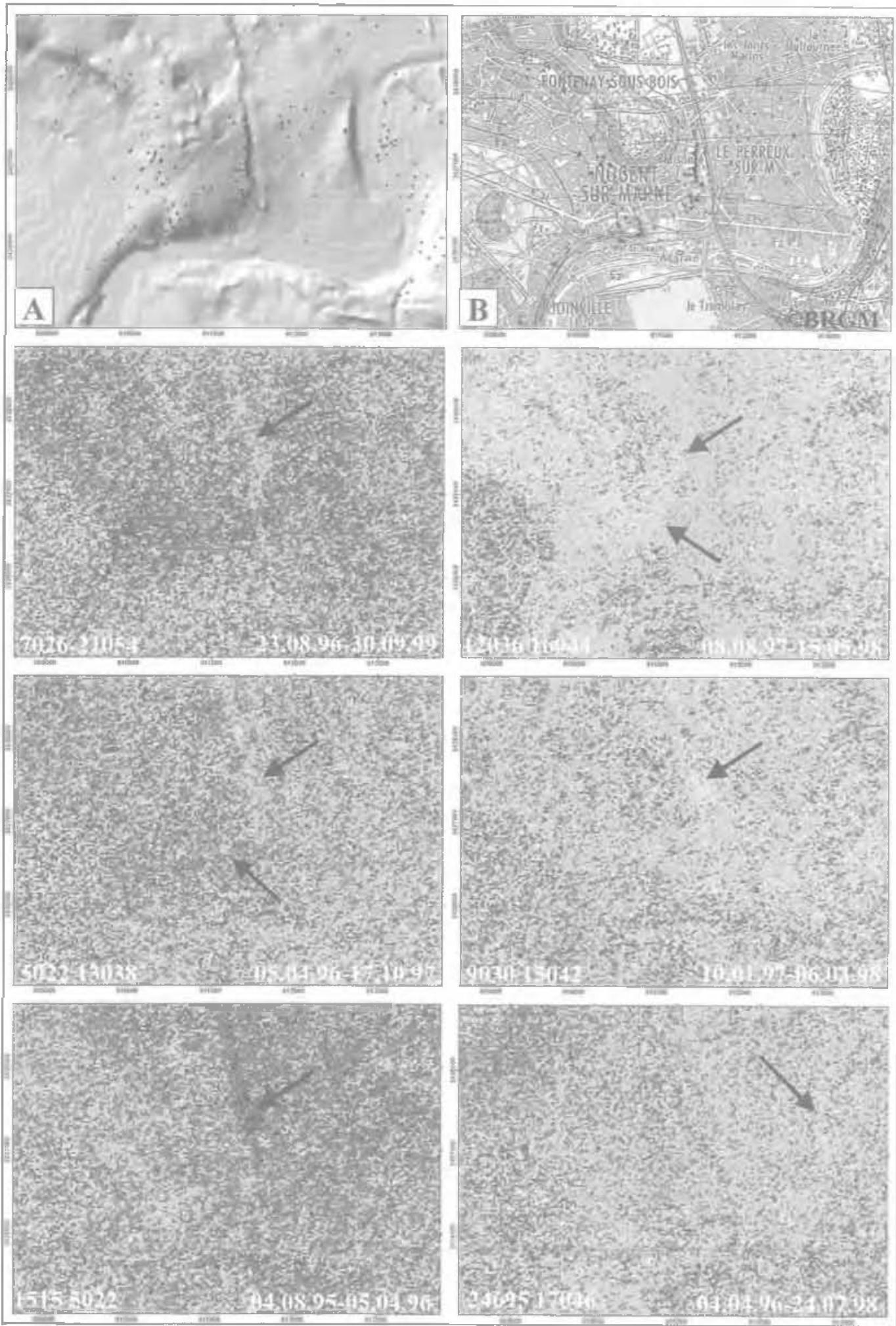

Plenche : Extraits d'interférogrammes sur la zone d'étude de Nogent-sur-Marne.

A- estompage de MNT révélant la topographie; (B) extrait de la carte géoloqique el de la sinistralité recensée par le BRGM (Donsimoni et al,, 2003). (disponible sur http:/www.univ-tnlv.fr/g2t/kaveh/). Extracts of interferogram on the study area of Nogent-Sur-Marne.

A- Hillshaded DTM revealing the topography; B- Extract of the geological map and damage identifed by the BRGM (Donsinori et al, 2003) (avaible on http//www.univ-mlvfr/g2i/kaveh/). 
spatiotemporelle du phénomène. Soulignons que les limitations d'usage sont nombreuses, du fait de la perte de corrélation géométrique, temporelle et des artefacts atmosphériques, qui limitent l'interprétation des anomalies dans uл milieu périurbain présentant beaucoup moins de réflecteurs que le milieu urbain plus dense. I semblerait que seules des déformations affectant une zone supérieurs à $1000 \mathrm{~m}^{2}$ sont potentiellement analysables par cette méthode.

Il s'avère donc nécessaire maintenant d'effectuer cette étude en utilisant une autre technique interférométrique, plus adaptée à des objets ponctuels de la taille des bâtiments : la méthode des points stables (PS), dénommé Persistent Scatterers interferometry [PSI] en anglais. L'interférométrie PSI, qui semble plus adaptée à la problématique du RGA permettrait de suive l'évo- lution temporelle de réflecteurs fortement rétrodiffusant pourvu que l'on précise la nature du réflecteur et qu'il soit suivi dans le temps avec une référence stable correctement localisée et déterminée.

\section{REMERCIEMENTS}

Ce travail s'inscrit dans le cadre du projet de recherche a aléa et risque sécheresse v de la fondation MAIF.

Nous tenons a remercier l'LAURIF pour avoir accondé l'utilisation du MOS 2003; la Fondation MAIF pour son aide financière; l'IGN pour les extraits de la BDortho sur l'Ile-de-France, issue du Geoportail ; le BRGM pour la carte géologique non vectorisée achetée, et la synthèse des données de sinistralité disponibles dans les rapports départementatx (cf. Www.argiles.ff). Nous tenons également à remercier vivement les relecteurs pour les améliorations apporteses au manuscrit.

\section{Bibliographie}

Bamler; R.r Hartl P. - Synthetic aperture radar interferometry. Inverse Problems 14(4), 1998, p. R1-R54.

Barbier P. - Comprendre le phénomêre de retrait/gonflement. Qualité Construction. $\mathrm{n}^{\circ} 87$. novembre-décembre 2004, p. 21-44.

Ben-Dor E., Patkin K., Banin A., Kamieli A. - Mapping of several soil properties using DAIS-7915 hyperspectral scanner data: A case study over clayey soils in Israel. International Journal of Remote Sensing, vol. $23, \mathrm{n}^{5} 6,30,2002$, p. $1043-$ 1062

Blgot G. Zerhouni M.I. - Retrait, gonflement et tassement des sols fins, Notes techniques. Bulletin des Laboratoires des ponts et chausses -229 , NT 4252,2000 , p. 105-114.

Bourrelier P... Deneufbourg G., De Vans say B. - Les Catastrophes naturelles, Editions OEM, 2000, 160p

Chabrillat S., Goetz A.F.H., Krosley L., Olgen H.W. - Use of hyperspectral images in the identification and mapping of expansive clay soils and the role of spatial resolution. Remote Sensing of Environment, vol. 82, Issues 2-3, 2002, p. $431-445$.

Donsimoni M. avec la collaboration de Clozier 1., Motteau M. Vincent M. - Cartographie de l'aléa retrat/gonflement des sols argileux dans le département du Val-de-Marne. BRGM/RP-52224-FR 2003, 5 fig., 16 tabi,, 2 ann., 4 pl, h.t.,
$133 \mathrm{p}$.

Donsimoni M., Kermeis M., Giraud F. avec la collaboration de Imbault M. et Le Roy S. - Cartographie de l'aléa retrait/gonflement des sols argileux dans le departement de Seine-et-Marne. Fapport BRGM/RP-53212-FR, 2006, 38 ill., 3 ann. 3 pl. h.t., 1 CD-Rom, $114 \mathrm{p}$.

Frmeau B., Deffontaines B., Rudant J.-P. Le Parmenter A-M. - Monitoring vertical deformation due to water pumping in the city of Paris (France) with differential interferometry. Comptes rendus Geosciences, vol. $33 \overline{7}$, Issue 13, 2005 . p. $1173-1183$

Hansen J. Sato M., Ruedy R., Lo K., Lea D.W. Medina-Elizade M. - Global temperature change. Proceedings of the National Acaderny of Sciences of the United States of America 006, 103, 1428814293 originally published online Sept 25, 2006; doi: 10.1073 /pnas.0606291103.

Hanssen, R.F. - Radar Interferometry: Data Interpretation and Error Analy5is. Remote Sensing and Digital image Processing, Kluwer Academic Publishers, Dordrecht. The Netherlands, 2001 . $308 \mathrm{p}$.

Kariuki P.C., Van der Meer F. - Issues of effectiveness in empirical methods for describing swelling soils. International Journal of Applied Earth Observation and Geoinformation, vol, 4. Issue 3, 2003, p. 231-241.

Kariuki P.C., Woldał T, Van der Meer F.
The Role of Remote Sensing in Mapping Swelling Soils. Asian Journal of Geoinformatics, vol. 5. $0^{\circ}$ 1, 2004, Published by ARSRIN, PO. Box 4, Klong Luang, Pathumthani 12120, Thailand.

Massonnet $\mathrm{D}_{,}$, Feigl K. - Radar interferom etry and its application to changes in the earth's surface. Review of Geophysics. vol. $36, n^{\circ} 4,1998$, p. $441-500$.

Pathier E. - Apports de l'interférométrie radar différentielte a l'étude de la tectonigue active de Taiwan. Thèse de l'université Marne-la-Vallée, [école doctorale ICMS), 2003, $272 \mathrm{p}$.

Suyer R., Lernoine M., Goguel J. - Carte gél. France (1/50 000), feuille Lagny, (type1922), $1^{\text {nt }}$ édítion, Orléans, BRGM, 1958.

Van der Meer F. -Can we map swelling clays with remote sensing? In: Van der Meer F., Nieuwenhuis G.J.A., Molenaar M. Woldai I. (eds.), Operational remote sensing for sustainable development. Journal of Applied Geoinformation Management (special section). 1(1). 1999. p. 27-35.

Vincent M. 2006. Prévention du risque séclłeresse : cartographie départernentale de l'alée retrait/gonflement des argiles et établissement de plans de prevention des risques. Revue géologue n $\mathrm{n}^{\circ} 146$, 2006, p. 43-47. 